\title{
Coordinated Control of Hybrid Energy Storage System in the Microgrid
}

\author{
Hua Li*, Yongfeng Ren, Le Li \\ School of electric power college, Inner Mongolia University of Technology, \\ Hohhot, Aimin street 49, 010051, China. \\ lihua0806@qq.com
}

\begin{abstract}
In allusion to the output power fluctuation of intermittent energy source in the wind-solar-micro-storage microgrid system, a coordinated control based on the hybrid energy storage system of microgrid and the power distribution between battery and super capacitor $(S C)$ is established. In view of the power management and energy distribution between the battery and SC two energy storage units, the output of fuzzy control of present charging/discharging capability of the hybrid energy storage system is utilized. Target power value is determined firstly, according to the battery and SC charged state synthetically, the deviation value beyond the target power value is allocated according to the fuzzy control theory between the two kinds of energy storage medium. The effectiveness of the proposed strategy is validated by results of the case study.
\end{abstract}

Keywords: Microgrid, Hybrid Energy Storage System, Coordinated Control

\section{Introduction}

The microgrid system is becoming an important development direction in many countries and regions along with our country economy development fleetly, electric power requirement advance year after year and energy sources \& environment contradiction looming large. Connecting the smart grid and energy internet is an effective approach to make the best of micro grid system[1-6]. However, the distributed renewable energy power generation intermittence and random characteristics restrict its power generation capability and its running stabilization[7-10]. The microgrid can join the distributed generation, burthen, energy storage equipment together through advanced control system, and form a controllable cell. It not only run with distribution power system connection grid, but also run without grid[11-15]. The microgrid connection may dig well the distributed generation and bring remarkable value into the power supply department and user[16-18].The hybrid storage system as the power tame unit of micro power supply and grid has a crucial role for the stable operation of the whole system and the effective use of power. ${ }^{1}$

In view of the power management and energy distribution between the battery and SC two energy storage units. Target power value is determined firstly, according to the battery and SC charged state synthetically, the deviation value beyond the target power value is allocated according to the fuzzy control theory between the two kinds of energy storage medium. The simulation results validated that hybrid storage system coordination control is viable and effective for the wind-solar-micro-storage microgrid system.

\section{Structure of the Wind-Solar- Micro-Storage Microgrid System}

The wind-solar-micro--storage microgrid system structure is shown as Figure 1. In Figure 1, the microgrid contains wind turbine (WT), photovoltaic (PV), micro turbine

$\mathrm{Hua} \mathrm{Li}$ is the corresponding author. 
(MT) and hybrid energy storage system. Microgrid is connected the medium voltage (MV) alternating current distribution network by AC/DC or DC/AC converters. Hybrid energy storage system is composed by SC and battery. Hybrid energy storage system is disposed at wind turbine and photovoltaic (PV) array export outlet for the power to stabilize the grid and islanding operation using a given voltage and frequency control. Meanwhile, the hybrid energy storage system can absorb or recharge the power shortfall of wind and solar in order to maintain the power balance of the entire system[19-21].Load1 and load2 are sensitivity load. It should guarantee the reliability of power supply when micro grid system operations. Load3 is the normal load, which can be removed when necessary.

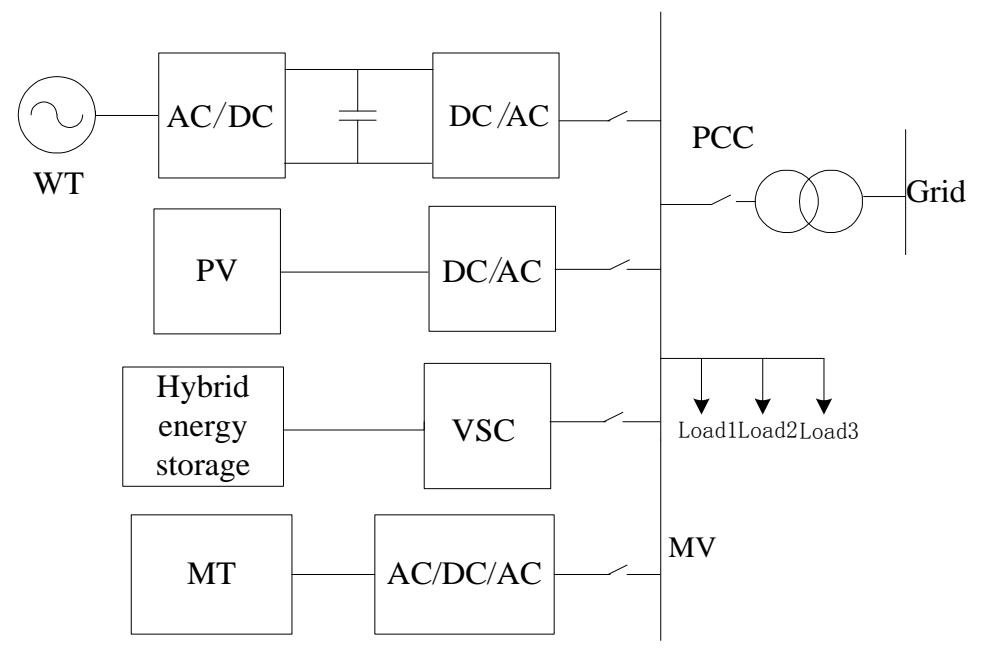

Figure 1. Wind-Solar-Micro-Storage Microgrid System Structure

\section{Coordinated Control Strategy of Hybrid Energy Storage System}

\subsection{SC and Battery Topology Structure}

The topology structure of SC and battery is shown as Figure 2. The battery power is controllable in this topology structure, which battery life is extend via optimizing the battery charge and discharge current. SC is varies according to the dc bus voltage. But the SC need series more units in order to obtain high voltages. In addition, the pulse load current range, SC terminal voltage will decline, but the inverter requires a relatively stable, normal work and the minimum voltage to generate the correct ac voltage, so dc voltage must be controlled within the appropriated range in order to effective operation.

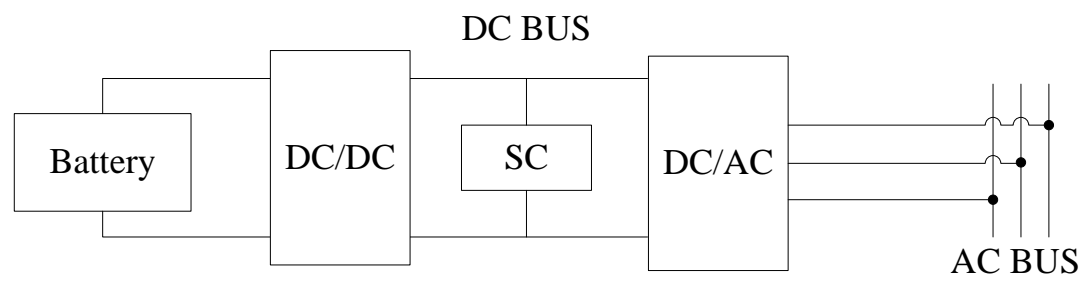

Figure 2. SC and Battery through the DC/DC Parallel Topological Structure

\subsection{Coordinated Control of SC and Battery}

Define $\Delta P(t)$ is smooth target external power value. The smooth target external power value is needed for the optimal power allocation between SC and battery. But the hybrid energy storage system is difficult to establish control model for charging and discharging 
accurately. In order to obtain the SOC when processing power allocation between SC and battery easily, the method based on fuzzy control theory is used for power allocation between SC and battery of energy storage system reasonably. The specific control optimization goal is as follows:

(1) The target external power is minimized after smoothing.

$\min \left|\triangle P(t)-P_{\text {HESS }}(t)\right|$

(2) In order to the power fluctuations is smoothed at next moment, SC is needed to save maximum energy.

$$
\min \left|S_{\text {soc_sc }}(t)-S_{\text {soc_sc }}(0)\right|
$$

(3) In order to the power fluctuations is smoothed at next moment, battery is needed to save maximum energy.

$$
\min \left|S_{s o c_{-} B}(t)-S_{s o c_{B} B}(0)\right|
$$

In the above formula, $P_{H E S S}(t)$ is the charge and discharge power of hybrid energy storage system at $t$ moment, the unit is $\mathrm{MW} ; S_{\text {soc } s c}(t), S_{s o c_{-} s c}(0)$ is the charged state(SOC) of SC at $t$ moment and initial moment respectively, $\% ; S_{s o c_{-} B}(t), S_{s o c_{-} B}(0)$ is the charged state of battery at $t$ moment and initial moment respectively, \%.

The optimize power allocation for SC and battery hybrid energy storage system should be combined with the size of the $S_{s o c_{-} s c}(t), S_{s o c_{-} B}(t)$ and $\Delta P(t)$. Two fuzzy control input $X_{1}(t)$ and $X_{2}(t)$ is set up in this paper:

$X_{2}(t)=S_{p d_{-} B}(t)-S_{s o c_{-} B}(0)$

$X_{1}(t)=S_{p d \_s c}(t)$

$X_{1}(t)$---- $S_{p d \_s c}(t) \quad$ (The SOC predicted value of the SC);

$X_{2}(t)$---- $S_{p d \_B}(t)-S_{\text {soc } \_B}(0) \quad$ (The SOC deviation predicted value of the Battery).

Figure 3 shows the membership function $X_{1}(t), X_{2}(t)$ and output membership function at $\Delta P(t) \geq 0$. Table 1 shows the fuzzy control rules used in the fuzzy control method. Figure 4 shows the input membership function of the $\mathrm{SC}$ at $\Delta P(t)<0$, the output membership function and the battery input membership function are the same as $\Delta P(t) \geq 0$.. Fuzzy control rules as shown in table 2 .

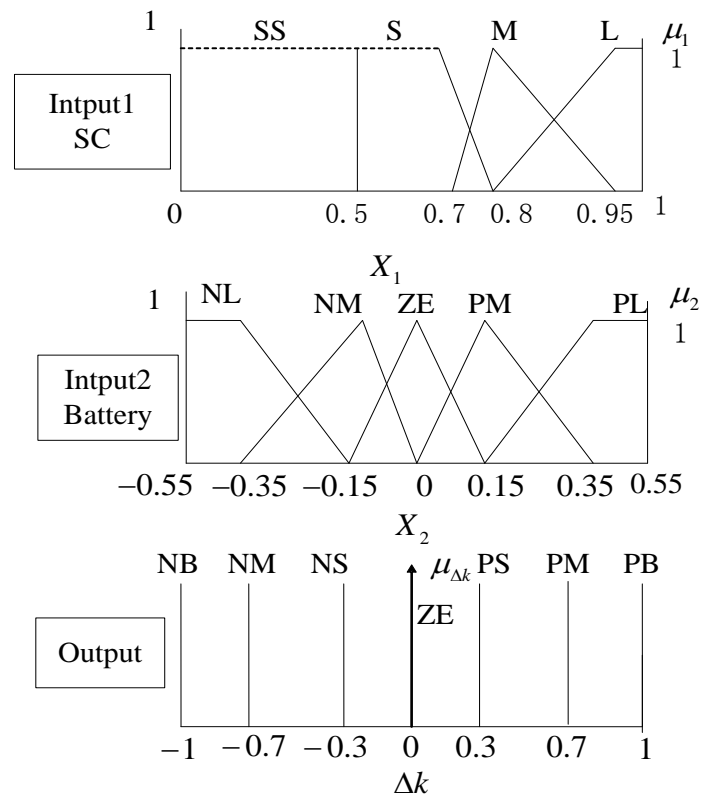

Figure 3. Membership Functions of $X 1(t), X 2(t)$ and Output $\Delta P(t) \geq 0$ 


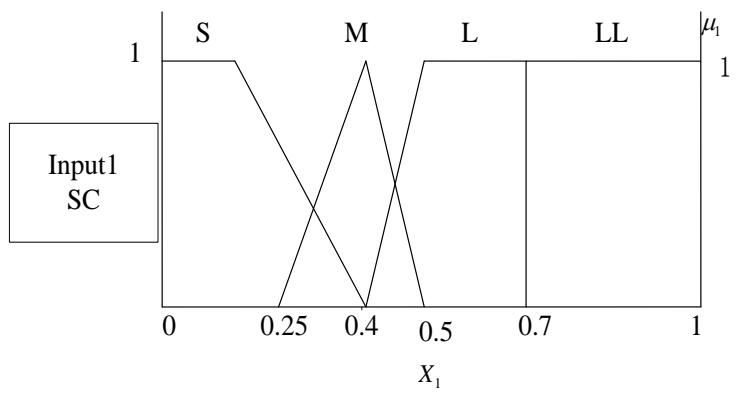

Figure 4. SC Input Membership Functions $\Delta P(t)<0$

Table 1. Fuzzy Control Rules at $\Delta \mathrm{P}(\mathrm{t}) \geq 0$

\begin{tabular}{cccccc}
\hline \multirow{2}{*}{$X_{1}(t)$} & \multicolumn{5}{c}{$X_{2}(t)$} \\
\cline { 2 - 6 } & $N L$ & $N M$ & $Z E$ & $P M$ & $P L$ \\
\hline$S S$ & $Z E$ & $Z E$ & $Z E$ & $Z E$ & $Z E$ \\
$S$ & $Z E$ & $Z E$ & $Z E$ & $Z E$ & $N S$ \\
$M$ & $N L$ & $N L$ & $N L$ & $N L$ & $N L$ \\
$L$ & $N M$ & $N L$ & $N L$ & $N L$ & $N L$ \\
\hline
\end{tabular}

Table 2. Fuzzy Control Rules at $\Delta \mathrm{P}(\mathrm{t})<0$

\begin{tabular}{cccccc}
\hline \multirow{2}{*}{$X_{1}(t)$} & \multicolumn{5}{c}{$X_{2}(t)$} \\
\cline { 2 - 5 } & $N L$ & $N M$ & $Z E$ & $P M$ & $P L$ \\
\hline$S$ & $P L$ & $P M$ & $P L$ & $P L$ & $P L$ \\
$M$ & $P M$ & $P S$ & $P L$ & $P L$ & $P L$ \\
$L$ & $Z E$ & $Z E$ & $Z E$ & $P S$ & $Z E$ \\
$L L$ & $Z E$ & $Z E$ & $Z E$ & $Z E$ & $Z E$ \\
\hline
\end{tabular}

The power correction coefficient $b(t)$ of SC and battery hybrid energy storage system is obtain at $t$ moment via making the solution fuzzy calculation for input $X_{1}(t)$ and $X_{2}(t)$ used weighted average method.

$b(t)=\frac{\sum_{i} \sum_{j} \mu_{1 i}\left(X_{1}(t)\right) \mu_{2 j}\left(X_{2}(t)\right) \Delta k_{i j}}{\sum_{j} \sum_{j} \mu_{1 i}\left(X_{1}(t)\right) \mu_{2 j}\left(X_{2}(t)\right)}$

In the above formula, $\mu_{l i}\left(X_{1}(t)\right)$ and $\mu_{2 j}\left(X_{2}(t)\right)$ is the membership value for the $i$ of $X_{1}(t)$ and the $j$ of $X_{2}(t)$. The set of $i$ is $\{S, M, L, L L\}$, the set of $j$ is $\{N L, N M, Z E, P M, P L\} . \Delta k_{i j}$ is the corresponding output value of input $\mu_{l i}(t)$ and $\mu_{2 j}(t)$.

$P_{s c_{-} r e f}(t), P O_{B_{-} r e f}(t)$, is the SC reference power and the battery reference power at $t$ moment, which can be described as

$P_{S C_{-} \text {ref }}(t)=\Delta P(t)+b(t)|\Delta P(t)|$

$P_{B_{-} \text {ref }}(t)=-b(t)|\Delta P(t)|$

The SC and battery hybrid energy storage system is sufficient capacity when $S_{s o c_{-} s c}(t) \leq 0.5$ after absorbing the power and $S_{s o c_{-} s c}(t) \geq 0.7$ after outputting the power, the other situation is not enough capacity. The concrete model of optimizing energy allocation for SC and battery hybrid energy storage system as

(1) If the SC has enough residual capacity, the output power of battery can be reduced. 
$\Delta P(t)$ is assumed by the $\mathrm{SC}$ independently. The $\mathrm{SOC}$ of $\mathrm{SC}$ can be recover soon, which make the tame results is better at next times.

(2) If the residual capacity of SC is insufficient, $\Delta P(t)$ is assumed by the SC and battery jointly. Which purpose is preventing the smooth results from affecting due to SOC energy out-of-limit of SC.

When $\quad \Delta P(t) \geq 0, \quad-1 \Delta P(t) \leq 0$; When $\quad \Delta P(t)<0,0<b(t) \leq 1 . \quad S_{s o c_{-} B}(t)$ increased after absorbing the power when battery has enough residual capacity, then battery will be distributed $\triangle P(t)$ less in order to preventing the smooth results from affecting due to SOC energy out-of-limit of battery. If $S_{s o c_{-} B}(t)$ is decreased after absorbing the power, then battery will be distributed the more target external power value, and the reference power of battery is increased meanwhile.

According to the energy out-of-limit problem, the power fluctuation is smoothed by SC when SC appearing energy out-of-limit state firstly. The power fluctuation is smoothed by the battery firstly When the battery occurring energy out-of-limit problem.

\section{Study System and Simulation Result}

\subsection{Simulation Parameter}

In the example, the WT rating Pwe $=21 \mathrm{~kW}$. The rated power of PV Ppve $=8 \mathrm{~kW}$. The MT rated power is $15 \mathrm{~kW}$.All the capacity of the battery and sc are $25 \mathrm{kw} . \mathrm{h}$. Sensitive load 1 and sensitive load 2 active power reference values are $16 \mathrm{kw}$ and $8 \mathrm{kw}$ respectively. The grid voltage is $380 \mathrm{~V}, 50 \mathrm{~Hz}$. Filtering inductance $\mathrm{L}=2 \mathrm{mH}$. Dc side capacitor $\mathrm{C}=220 \mu \mathrm{F}$. Dc side voltage $\mathrm{E}=600 \mathrm{~V}$. Power supply equivalent resistance value $\mathrm{R}=0.1 \Omega$.Line parameter $R / X=0.642 / 0.083 \Omega / \mathrm{km}$.

\subsection{Simulation Result}

Figure 5 shows the wind speed change state of WT in this microgrid system, the wind speed $v$ is changed up and down based on the rated wind speed $12 \mathrm{~m} / \mathrm{s}$. And Figure 6 is the light and temperature waveform of solar micro power supply.

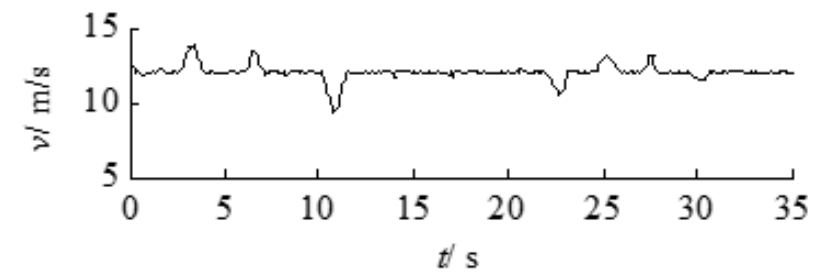

Figure 5. The Diagram of Wind Speed Change
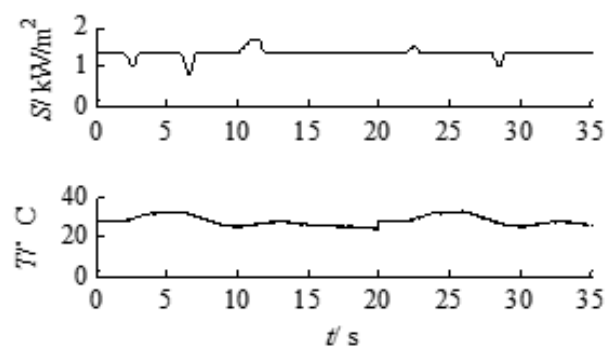

Figure 6. The Light and Temperature Diagram of Solar

Wind-solar-micro-storage microgrid system is operated the connected grid state before one second, then it is run island model after with the grid disconnection at one second 
time. Microgrid connected to the grid again at twenty second, the corresponding simulation results as shown in the figure below. Figure 7 shows the output active power of four micro power sources, PW is the output active power of wind turbine, PPV is the photovoltaic output active power, $\mathrm{Pb}$ is the output active power of battery and Psc is the output active power of SC. Figure 7 shows the battery can stabilize the wind power and photovoltaic fluctuations very well. In Fig.8, the output reactive power of four micro power sources, QW is the output reactive power of wind turbine, QPV is the photovoltaic output reactive power, $\mathrm{Qb}$ is the output reactive power of battery and Qsc is the output active power of SC.

Figure 5- Figure 7 shows the microgrid system got wind energy and light energy in the form of maximum power tracking as the change of wind speed and illumination, and dynamic response is rapidly. The microgrid operated in island model at $2 \mathrm{~s}-20 \mathrm{~s}$ times. The power after the wind and light supplement is averaged via battery and SC with the increase or decrease of wind speed and illumination. The hybrid energy storage system charged and discharged is reasonable according to different situations in order to maintain the power balance of whole microgrid system. Meanwhile, the reactive power of load need is provided by the hybrid energy storage system overall. Because of the microgrid is disconnected with grid, the microgrid is not provided reactive power by the power grid system, and the reactive power from the hybrid energy storage system is increased at this time. So, the reactive power from the wind and photovoltaic array are configured zero value for ensuring maximum utilization of wind and light energy, the simulation results shown in Figure 8.

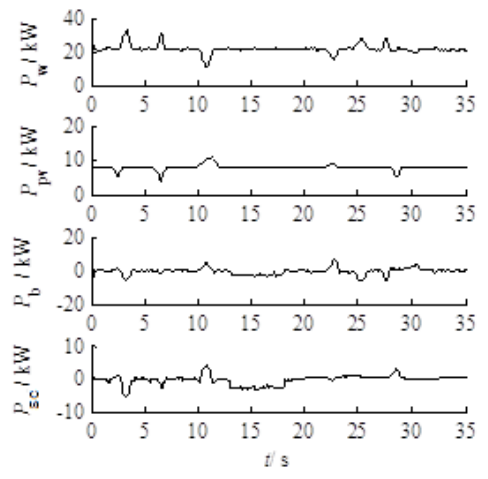

Figure 7. The Output Active Power Simulation Waveform of Micro Source

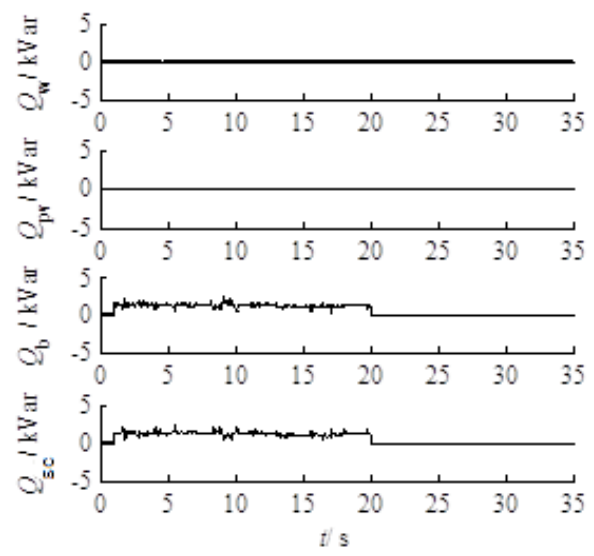

Figure 8. The Output Reactive Power Simulation Waveform of Micro Source 
The micro grid changed as connected grid operation again at 21s-35s times, and hybrid energy storage system adopted constant power control at this time. Wind power Pwg and photovoltaic power $\mathrm{P}_{\mathrm{pvg}}$ transmitted according to the power after filtering under the action of batteries, the waveform as shown in Figure 9 and Figure 10. That is to say the battery and SC can stabilize the wind power and photovoltaic fluctuations very well. In the process of the whole model transformation, system power changes gently, without the big power rush impacted on power grid, shown the good transient characteristics.

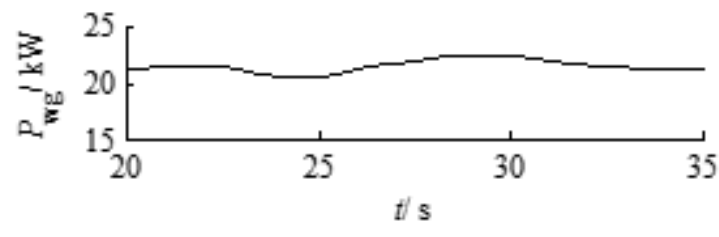

Figure 9. Wind Power Waveform after Stabilizing

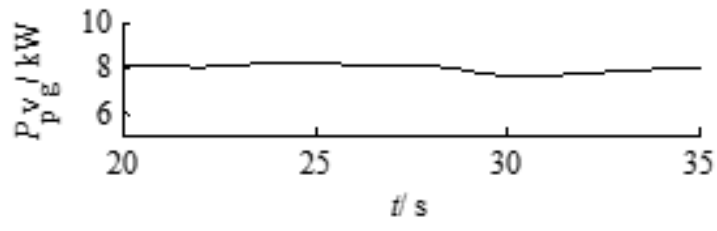

Figure 10. PV Power Waveform after Stabilizing

Figure 11 shows the voltage of Load1, Load2 is almost unchanged, that indicate microgrid can make important load is continuous and reliable power supply in the whole running time. The normal Load3 is removed when the microgrid system is running at13s 18s, Figure 12 and Figure 13 show the DG output power of active and reactive power will follow decreases with the decrease of the load respectively. Which illustrate the coordination control mode can adjust power timely in order to reach a new stable running state.

The load active total power and reactive total power waveform is shown in Figure 14 and Figure 15 respectively. Figure 16-Figure 17 shows the active power and reactive power of distribution network transmission. Combined with Figure 14- Figure 17, the microgrid system is stable operation and the reactive power of each DG is provided by power grid when the microgrid system in the connect grid running. In parallel operation mode, the output active power of microgrid can satisfy the load demand, and the excess or lack part power is assumed via the power grid when power fluctuation state. At the same time by the charts can also be learned in parallel operation mode, each DG reactive power output is zero, which indicates the load needed all the reactive power is provided though distribution network .
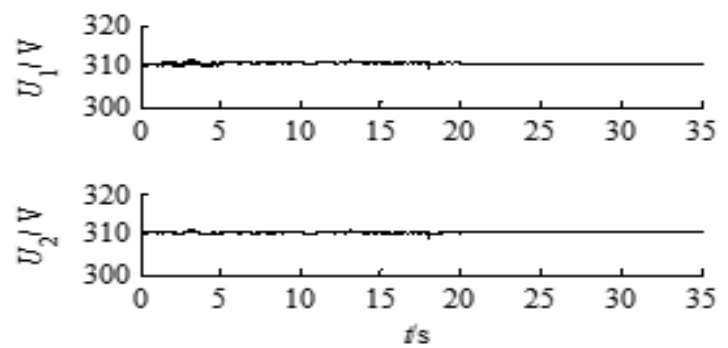

Figure 11. Load1, Load2 Voltage Waveform 


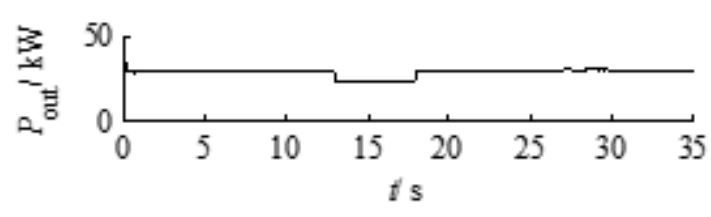

Figure 12. DG Active Power Sum Waveform

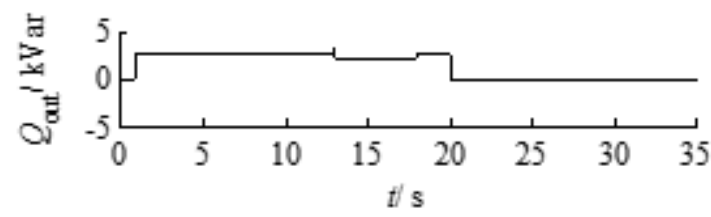

Figure 13. DG Reactive Power Sum Waveform

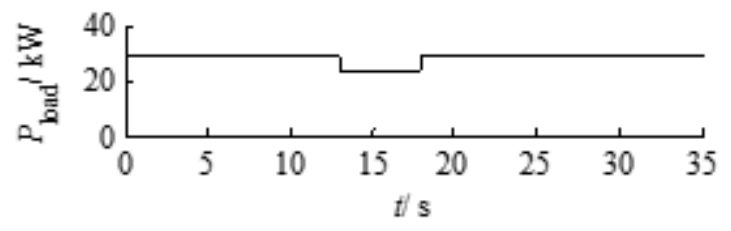

Figure 14. Load Active Power Sum Waveform

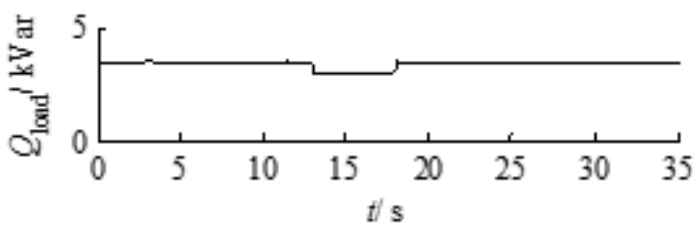

Figure 15. Load Reactive Power Sum Waveform

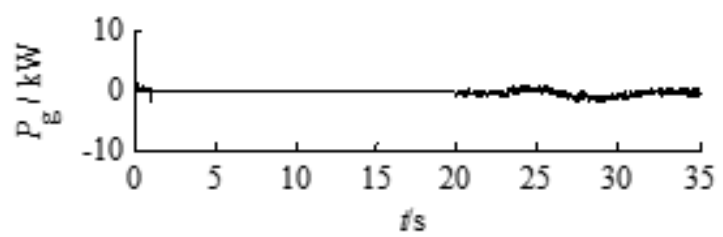

Figure 16. Distribution Network Transmission Active Power

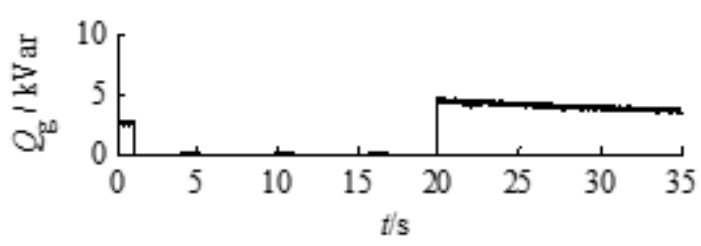

Figure 17. Distribution Network Transmission Reactive Power 


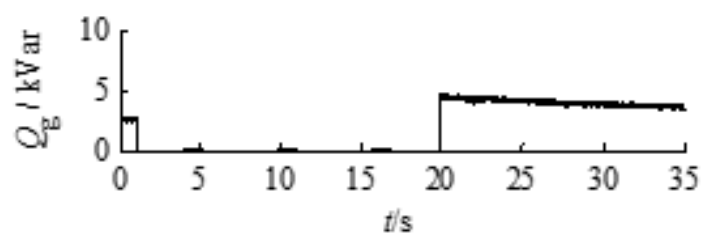

Figure 18. System Frequency Waveform

In Figure 18, the system frequency is almost remain $50 H z$ the same, that is to say the microgrid system can maintain operation on reference frequency value whether to switch from the operation mode or increase or decrease the load. That verified the feasibility and the superiority of the coordinate control strategy based on the battery and SC hybrid energy storage system.

\section{Conclusions}

This study make the fuzzy coordinated control hybrid energy storage system apply to wind-solar-micro-storage microgrid system. In view of the power management and energy distribution between the battery and super capacitor two energy storage units. Target power value is determined firstly, according to the battery and super capacitor charged state synthetically, the deviation value beyond the target power value is allocated according to the fuzzy control theory between the two kinds of energy storage medium. The theoretical analysis and simulation research validate this method can stabilize the wind power and photovoltaic fluctuations very well. These controls let the wind-solar-micro storage microgrid system more conducive to realize the real time control and improve the efficiency of renewable energy in microgrid system.

\section{Acknowledgements}

This paper comes from the hybrid microgrid comprehensive coordinated control and energy allocation strategy research of Inner Mongolia natural science foundation 2016BS0508.

\section{References}

[1] Christopher M. Colson, M. Hashem Nehrir, Ratnesh K. Sharma, et al., J. Improving Sustainability of Hybrid Energy Systems Part II: Managing Multiple Objectives With a Multiagent System. IEEE TRANSACTIONS ON SUSTAINABLE ENERGY. 5, 1 (2014)

[2] Menniti, D., Sorrentino, N., Pinnarelli, A., et al., J. In the future Smart Cities: Coordination of micro Smart Grids in a Virtual Energy District. Power Electronics, Electrical Drives, Automation and Motion (SPEEDAM). 2, 1 (2014)

[3] Van Berkel, K.,Titulaer, R. ,Hofman, T. , et al. , J. From Optimal to Real-Time Control of a Mechanical Hybrid Powertrain. IEEE Transactions on Control Systems Technolog. 23, 2 (2015)

[4] Graditi, G. , Di Silvestre, M.L. ,Gallea, R. , et al. , J. Heuristic-Based Shiftable Loads Optimal Management in Smart Micro-Grids. IEEE Transactions on Industrial Informatics. 11, 11 (2015)

[5] Zina Boussaada, Octavian Curea, Haritza Camblong, et al. J. Multi-agent systems for the dependability and safety of microgrids. Int J Interact Des Manuf Int J Interact Des Manuf. 30, (2014)

[6] Eghtedarpour, N, Farjah , E. , J. Distributed charge/discharge control of energy storages in a renewable-energy-based DC micro-grid. Renewable Power Generation, IET.. 8, 1 (2014)

[7] Wang Xingang, Guzhen, Zang Gewei, et a. 1, J. Containing multiple coordinated control of the distributed power grid. East China electric power. 42, 11 (2014)

[8] Yangyang, Caixu, J. The simulation study of wind-solar-storage micro grid coordinate control base on the RTDS scenery. Renewable energy. 32, 2 (2014)

[9] Binayak Bhandari, Kyung-Tae Lee, Gil-Yong Lee, et al., J. Optimization of hybrid renewable energy power systems: A review. International Journal of Precision Engineering and Manufacturing-Green Technology. 2, 1 (2015) 
[10] Camblong Haritza, Curea Octavian, Etxeberria Aitor, et al., J. Research experimental platforms to study microgrids issues. Int J Interact Des Manuf. 31, (2015)

[11] Sohrab Mirsaeidi, Dalila Mat Said, Mohammad Wazir Mustafa, et al., J. Design and testing of a centralized protection scheme for micro-grids. Cent. South Univ. 22, (2015)

[12] V. Pavan Kumar and Ravikumar Bhimasingu, J. Renewable energy based microgrid system sizing and energy management for green buildings. Mod. Power Syst. Clean Energy. 3, 1 (2015)

[13] Konstantinos O. Oureilidis, Emmanouil A. Bakirtzis and Charis S. Demoulias, J. Frequency-based control of islanded microgrid with renewable energy sources and energy storage. Mod. Power Syst. Clean Energy. 4, 1 (2015)

[14] Pedro Crespo Del Granado, Stein W. Wallace and Zhan Pang, J. The impact of wind uncertainty on the strategic valuation of distributed electricity storage. Comput Manag Sci.. 13, (2015)

[15] Pamela Manjarres and Om Malik, J. Frequency regulation by fuzzy and binary control in a hybrid islanded microgrid. Mod. Power Syst. Clean Energy. 3, 3 (2015)

[16] Abdalkarim Awad, Peter Bazan and Reinhard German, J. Optimized operation of PV/T and micro-CHP hybrid power systems. Technol Econ Smart Grids Sustain Energy. 23,(2016)

[17] Guangqian Ding, Ran Wei, Ke Zhou, et al., J. Communication-less harmonic compensation in a multi-bus microgrid through autonomous control of distributed generation grid-interfacing converters. Mod. Power Syst. Clean Energy. 3, 4 (2015)

[18] Kubilay Demir, Daniel Germanus and Neeraj Suri. J. Robust QoS-aware communication in the smart distribution grid. Peer-to-Peer Netw. Appl.. 24, (2015)

[19] An Luo, Qianming Xu, Fujun Ma, et al., "Overview of power quality analysis and control technology for the smart grid," J. Mod. Power Syst. Clean Energy. 4, 1 (2016)

[20] Gabriella Ferruzzi, Giorgio Graditi, Federico Rossi, et al., J. Optimal operation of a residential microgrid: The role of demand side management. Intell Ind Syst. 1, (2015)

[21] Thongchart Kerdphol, Yaser Qudaih, Masayuki Watanabe, et al., J. RBF neural network-based online intelligent management of a battery energy storage system for stand-alone microgrids. Kerdphol et al. Energy, Sustainability and Society. 6, 5 (2016) 